\title{
ACOUSTIC ASPECTS OF ACTIVE-TWIST ROTOR CONTROL
}

\author{
Earl R. Booth, Jr. \\ e.r.booth@larc.nasa.gov \\ Matthew L. Wilbur \\ Aeroacoustics Branch, AAAC \\ m.1.wilbur@larc.nasa.gov \\ Army Research Laboratory \\ NASA Langley Research Center \\ Hampton, VA, 23681
}

\begin{abstract}
The use of an Active Twist Rotor system to provide both vibration reduction and performance enhancement has been explored in recent analytical and experimental studies. Effects of activetwist control on rotor noise, however, had not been determined. During a recent wind tunnel test of an active-twist rotor system, a set of acoustic measurements were obtained to assess the effects of active-twist control on noise produced by the rotor, especially blade-vortex interaction (BVI) noise. It was found that for rotor operating conditions where BVI noise is dominant, active-twist control provided a reduction in BVI noise level. This BVI noise reduction was almost, but not quite, as large as that obtained in a similar test using HHC. However, vibration levels were usually adversely affected at operating conditions favoring minimum BVI noise. Conversely, operating conditions favoring minimum vibration levels affected BVI noise levels, but not always adversely.
\end{abstract}

\section{Notation}

BVISPL bandpass acoustic level integrated from $6.5 \mathrm{f}_{\mathrm{bp}}$ to $40.5 \mathrm{f}_{\mathrm{bp}}, \mathrm{dB}$

$\mathrm{f}_{\mathrm{bp}} \quad$ blade passage frequency, $\mathrm{Hz}$

LFSPL bandpass acoustic level integrated from $0.5 \mathrm{f}_{\mathrm{bp}}$ to $6.5 \mathrm{f}_{\mathrm{bp}}, \mathrm{dB}$

NF fixed-system vertical shear force, aligned with the rotor shaft, $\mathrm{lb}$

$\mathrm{nP} \quad \mathrm{n}$ cycles per rotor revolution, i.e. $5 \mathrm{P}$

$\mathrm{q} \quad$ tunnel dynamic pressure, $\mathrm{lb} / \mathrm{ft}^{2}$

$\mathrm{R} \quad$ rotor radius, 55 in

SPL sound pressure level, $\mathrm{dB}$

$\mathrm{V}$ tunnel velocity, $\mathrm{ft} / \mathrm{s}$

w/q data scatter parameter, $\mathrm{Pa}$

$\mathrm{X}$ distance downstream of hub center, in

$\mathrm{Y}$ distance to port side of hub center, in

$\mathrm{Z} \quad$ distance above hub center, in

$\alpha \quad$ rotor shaft angle of attack, deg

$\mu \quad$ advance ratio, $\mathrm{V} / \mathrm{R} \Omega$

$\Omega \quad$ rotor rotational speed, radians/s

\section{Introduction}

Rotorcraft noise and vibration reduction are two primary thrusts of current rotorcraft research. Noise reduction research has explored the use of both passive and active means of reducing rotor noise. Most active noise reduction technologies were originally intended as vibration reduction technologies and were subsequently shown to be effective in noise reduction as well. Three examples of such technology are higher harmonic control $^{1-3}$, individual blade control ${ }^{4}$, and active rotor flaps 5 . In general, active noise control methodologies have exhibited loss of vibration control effectiveness for control inputs that reduce noise, and vice versa. Also, some of the noise reduction methods tend to increase some types of rotor noise in order to decrease other types of noise. For example, it was found ${ }^{1,2}$ that $\mathrm{HHC}$ reduced $\mathrm{BVI}$ noise, but increased low frequency noise.

Recent progress in active-twist technology for vibration reduction and performance improvement made activetwist rotor control an attractive candidate to examine as a potential noise reduction technology. This work is the first exploration of active-twist rotor control to reduce helicopter BVI noise. The current results are from a previously reported test ${ }^{6}$ to explore vibration reduction in which simultaneous acoustic and vibration measurements were obtained for a large number of operating conditions. The current work examines noise and vibration results to determine the extent to which active-twist control meets the goal of comprehensive rotor noise and vibration control. 


\section{Apparatus and Data Acquisition}

\section{Wind Tunnel}

The Langley Transonic Dynamics Tunnel is a continuous-flow pressure tunnel capable of speeds up to Mach 1.2 at stagnation pressures up to $1 \mathrm{~atm}$. The TDT has a $16-\mathrm{ft}$ square slotted test section that has cropped corners and a cross-sectional area of $248 \mathrm{ft}^{2}$. Either air or R-134a, a heavy gas, may be used as the test medium. Forward-flight testing of the ATR was conducted in the heavy gas test medium at a constant density of 0.0047 $\mathrm{sl} / \mathrm{ft}^{3}$.

\section{Model Description}

Testbed. The Aeroelastic Rotor Experimental System (ARES) helicopter testbed, shown in figures 1 and 2, was used for all forward-flight testing. The ARES is powered by a variable-frequency synchronous motor rated at 47 -hp output at $12,000 \mathrm{rpm}$. The motor is connected to the rotor shaft through a belt-driven, twostage speed-reduction system. Rotor control is achieved by a conventional hydraulically-actuated rise-and-fall swashplate using three independent actuators. Similarly, rotor-shaft angle of attack is controlled by a single hydraulic actuator.

A six-component strain-gage balance placed in the fixed system 21.0 inches below the rotor hub measures rotor forces and moments. The strain-gage balance supports the rotor pylon and drive system, pitches with the model shaft, and measures all of the fixed-system forces and moments generated by the rotor model. A streamlined fuselage shape encloses the rotor controls and drive system; however, the fuselage shape is isolated from the rotor system such that its forces and moments do not contribute to the loads measured by the balance.

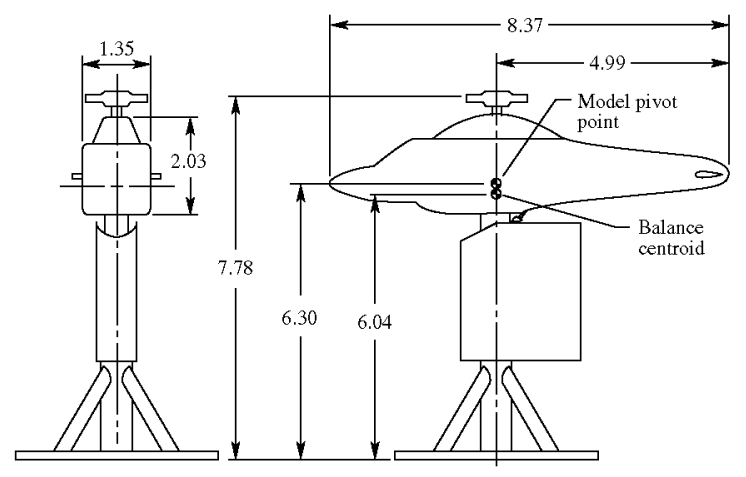

Figure 1. Schematic of the Aeroelastic Rotor Experimental System (ARES) helicopter testbed. All dimensions are in feet.
Figure 2 shows the Active Twist Rotor mounted on the ARES helicopter testbed in the TDT. For this configuration a four-bladed articulated hub with coincident flap and lag hinges is used on the ARES. coupling is minimized. A more detailed discussion of the ARES testbed can be found in reference 6 .

ATR Blades. Active fiber composite (AFC) actuators are used to twist the ATR rotor blades. In figure 3, the $\mathrm{AFC}$ actuators are shown in conceptually and as the actuator package used in the blade construction. Each ATR blade uses 24 actuators, as shown in the figure, embedded directly in the structure of each blade D-spar, spanning from $0.30 \mathrm{R}$ ( $30 \%$ blade radius) to $0.98 \mathrm{R}$. The actuators are placed in four layers through the thickness of the blades and are oriented such that the active strain is applied at $\pm 45^{\circ}$ relative the blade spanwise axis to permit maximum torsional control of the blades. Actuation of the AFCs is accomplished using separate high-voltage, low-current power channels for each blade. The resulting torsion applied to the blade results in a controllable twist of the blade. The amplitude of this blade twist at the blade tip was measured in forward flight conditions using projection moiré interferometry ${ }^{7}$ (PMI) and was found ${ }^{6}$ to be 1.1 degrees at $3 \mathrm{P}, 1.3$ degrees at $4 \mathrm{P}$ and 1.4 degrees at $5 \mathrm{P}$ for an actuation amplitude of 1000 volts. In addition, at a frequency of $3 \mathrm{P}$, blade tip twist amplitude was found to be 0.5 and 0.8 degrees at 500 and 750 volts actuation amplitudes, respectively.

The ATR blades have a rectangular planform with a chord of 4.24 inches, radius of 55.0 inches, and a NACA-0012 airfoil section. Pretwist is linear with a twist of $-10^{\circ}$ from the center of rotation to the blade tip.

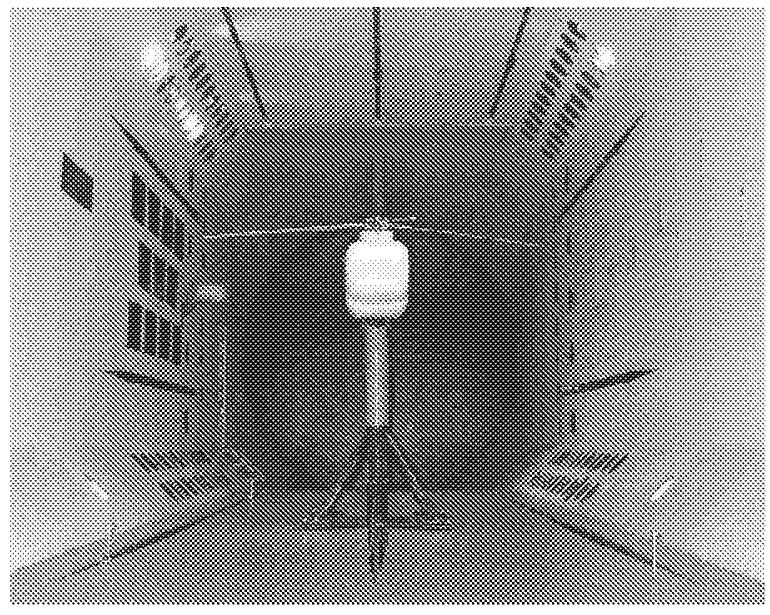

Figure 2. ARES with ATR rotor hardware mounted in the TDT test section with microphones installed. 


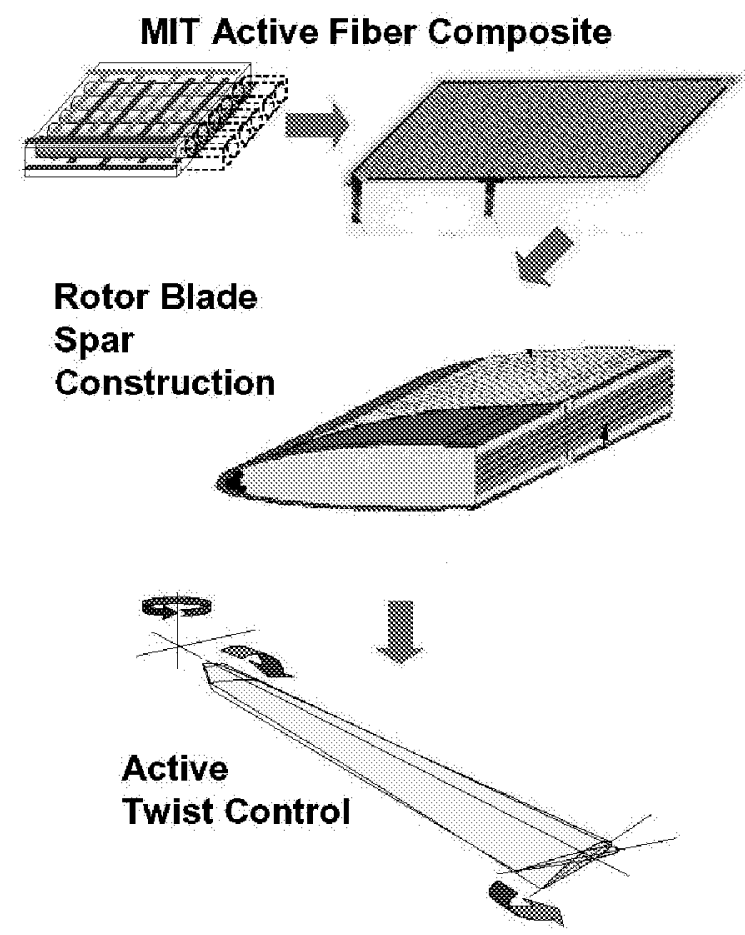

Figure 3. Active-twist concept.

ATR Computer Control System. Active-twist control of the ATR blades is achieved with a computer control system incorporating a digital signal processor board, 32 analog-to-digital channels, 6 digital-to-analog channels, and 32 digital input-output channels. Available control types include a collective twist mode where all four blade are twisted simultaneously, and an Individual Blade Control (IBC) mode where each blade twists according to a prescribed schedule associated with its position in the rotor azimuth. IBC mode control was used for the current investigation. For each operating

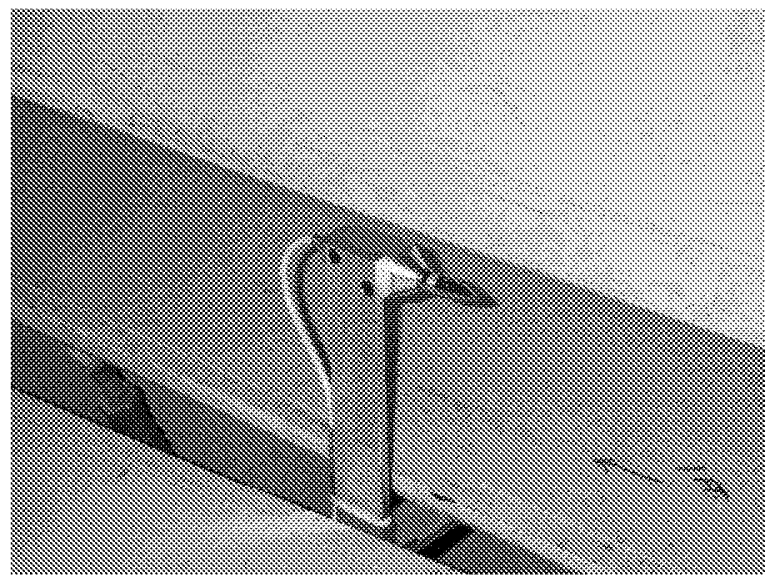

Figure 4. Close up view of fixed microphone holder. mode the ATR computer control system generates lowvoltage, sinusoidal control signals for each blade that are amplified by linear high-voltage amplifiers to a voltage amplitude of 1000 volts, approximately one-half of the $\mathrm{AFC}$ maximum design voltage.

Acoustic Instrumentation. Acoustic data were acquired using six B\&K 4134 half-inch microphones fitted with B\&K UA 0386 streamlined nosecones. The microphones were mounted in fixed, minimally intrusive, microphones holders. Three microphones were mounted upstream of the model and three were mounted downstream of the model. Location data for the microphones is presented in table 1. Four of the microphones are visible in figure 2, two mounted on the floor upstream of the model, one mounted on the floor downstream of the model and one mounted on the right side wall. A close up view of one of the microphones installed in the tunnel is shown in figure 4.

\section{Data Acquisition}

ARES ATR Data Acquisition. Data acquisition throughout the test was accomplished using multiple separate data acquisition systems and the ATR computer control system. The primary system used for acquisition of the vibratory loads data was a Modcomp computer with a Neff 600-series, 256-channel, 16-bit analog-to-digital converter with sample-and-hold. Lowpass anti-aliasing filters were set to $200 \mathrm{~Hz}$ for each data channel and a sampling rate of 1000 samples-persecond was used. Five seconds of data were typically acquired on the Modcomp computer system for each data point. A subset of the channels processed by the Modcomp system was also sampled by the ATR computer control system. The sampling rate on this system was 4000 samples-per-second, with data acquired for 3 seconds for each data point. Generally, the ATR computer control system was used to initiate data acquisition streams on each of the systems simultaneously.

Acoustic Data Acquisition. The acoustic data acquisition system was triggered by the ATR control system for each data point. The six microphone signals

Table 1. Microphone placement data.

\begin{tabular}{cccc}
\hline \hline Microphone & $\mathrm{X}$, in & $\mathrm{Y}$, in & $\mathrm{Z}$, in \\
\hline 1 & -99.5 & -58.8 & -89.5 \\
2 & -113.8 & 59.0 & -89.5 \\
3 & -113.8 & 59.0 & 89.5 \\
4 & 174.8 & -59.3 & -89.5 \\
5 & 183.5 & -88.5 & -47.5 \\
6 & 205.0 & -58.8 & 89.5 \\
\hline \hline
\end{tabular}


were each sampled at 1024 samples per rotor revolution (nominal sample rate of approximately 11,700 samples per second), anti-alias filtered at $5 \mathrm{kHz}$, for 60 rotor revolutions, yielding a sample time of slightly more than 5 seconds.

\section{Test Procedures}

All testing was conducted in the heavy gas test medium of the TDT at a nominal density of $0.0047 \mathrm{sl} / \mathrm{ft}^{3}$. The rotor rotational speed throughout the test was held at a constant $688 \mathrm{rpm}$, resulting in a nominal hover tip Mach number of 0.60 . The bulk of the testing was conducted in forward flight with various steady-state trim conditions representative of sustained $1 \mathrm{~g}$ level flight and descending flight. A rotor lift coefficient of 0.0066 was chosen for the nominal lifting task throughout the test, and the rotor-shaft angle of attack was chosen as a function of flight speed to represent the various flight conditions.

\section{Data Analysis}

Data Quality. The TDT is a hard wall wind tunnel, and has only rarely been used to make acoustic measurements. In a previous study ${ }^{1}$, it was shown that sound power measurements can me made in this tunnel to assess rotor noise reduction. Subsequent testing ${ }^{2,3}$ in anechoic wind tunnels have borne out the results from that test.

The hard wall tunnel test section can be regarded to be reverberant except for two surfaces through which sound power can escape the test section, namely the tunnel cross sections upstream and downstream of the model. If a control volume is drawn around the model in the test section such that four faces of the box are at the tunnel walls, then the remaining two faces become the two control surfaces through which acoustic energy can escape. Thus the sound power produced by the model should be equal to the sound power flux across these two surfaces. All that is required is to estimate the sound flux upstream and downstream of the model.

Accordingly, for this test, the microphones were mounted in the test section, half upstream of the model and half downstream of the model, using short lowintrusion microphone holders. These low-intrusion microphone holders were desired for this test to minimize interference with the active-twist control vibration results. While the microphones upstream of the model worked well, the downstream microphones were adversely affected by test section wall boundary layers and separated flow. Due to the importance of this data quality issue to the results of this study, it is worth examination in detail. The fundamental question was which microphone signals were good and which were bad?

In order to investigate this data quality issue, the acoustic data were post-test processed to obtain average time history data. Data were averaged in blocks of 1024 points (one revolution) of data for 60 revolutions of data. In addition, the minimum and maximum values at each time step were recorded. The resulting data consisted of an average time history with a minimum and maximum boundary. As an example, time history data from a baseline condition (no active-twist control active) at $\mu=0.20$ and $\alpha=1.0$ degree, are shown in figure 5. Microphone 1 is one of the three microphones located upstream, while microphone 6 is located downstream of the model on the tunnel ceiling. The data scatter for microphone 1 is small, showing this signal to be acceptably steady for an in-flow microphone, while data scatter (note the scale change) for microphone 6 is not acceptable. The magnitude of

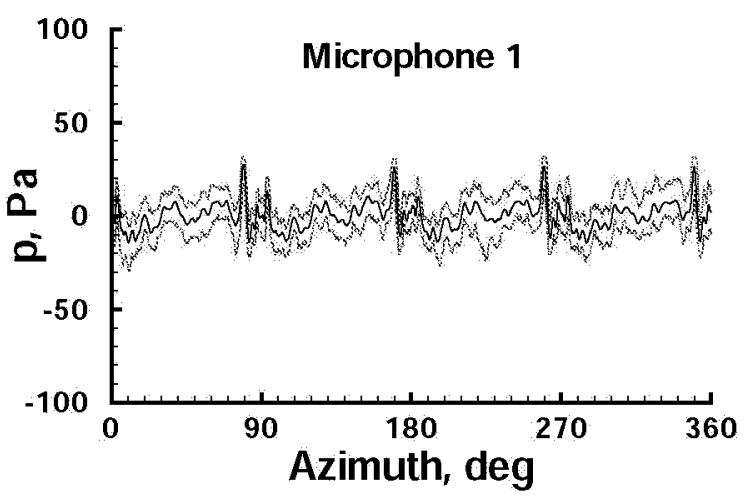

(a) Time history data from microphone 1.

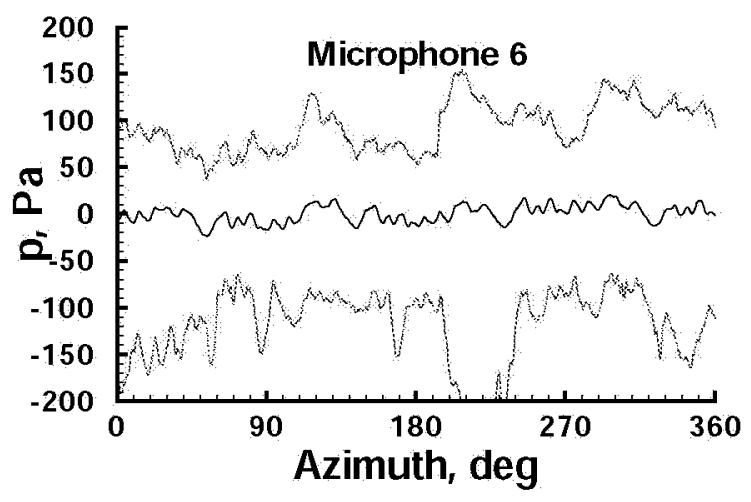

(b) Time history data from microphone 6 .

Figure 5. Averaged time history for two microphone signals showing minimum and maximum data scatter envelope for $\mu=0.20$ and $\alpha=1 \mathrm{deg}$. 
the scatter for microphone 6 is indicative of nonacoustic pressure fluctuations resulting from unsteady flow, possibly due to separation of flow from the tunnel surface adjacent to the microphone location. The low frequency energy from these large non-acoustic pressure fluctuations tends to saturate the microphone signal. In addition, the unsteady flow results in a variable signal path from the rotor to the microphone, which smears the signal. These effects combine to render the measurement unusable.

The first step to resolving a problem is to admit that the problem exists. The next step is to determine the extent of the problem. The means used in the current study to determine the extent of this data quality problem was to define a data scatter parameter and then use that parameter to separate the good data measurements from the unacceptable data measurements.

In figure 5 , the difference between the maximum and minimum values for each time step defines an envelope width, w, which is in units of pressure.

$\mathrm{w}(\mathrm{t})=\operatorname{maximum}(\mathrm{t})-\operatorname{minimum}(\mathrm{t})$

The value of $w(t)$ is averaged over the entire time period to produce a single parameter, $\mathrm{w}$, that characterizes data scatter for that data measurement. The value of $w$ was then non-dimensionalized by the tunnel dynamic pressure, q, and the resulting parameter was used to sort the measurements based on signal quality. If data scatter is primarily due to acoustic variability, as is suggested to be the case for microphone 1 as shown in figure 5(a), then the value of w/q should decrease with increasing speed. If data scatter is due to flow-related, but non-acoustic pressure fluctuations, as appears to be the case for microphone 6 in figure 5(b), then the value of w/q will both be a larger value and will either increase or tend to remain somewhat constant with increasing flow velocity.

The scatter parameter w/q was calculated for each microphone at each test point. The resulting data is shown in figure 6(a) for microphone 1, which is also representative of the results for the other two upstream microphones. For microphone $1, \mathrm{w} / \mathrm{q}$ is shown to be less than 0.10 at all conditions and the value decreases as tunnel speed increases. This indicates that the upstream microphone measurements are good throughout the test matrix. However, since acoustic flux needs to be estimated on both the upstream and downstream control surfaces, this result, while good news, only solves half the problem. In figure 6(b), the scatter data for microphone 4 shows a marginally higher value for $w / q$ at $\mu=0.14$ than was shown for microphone 1 in figure 6(a), which indicates that this

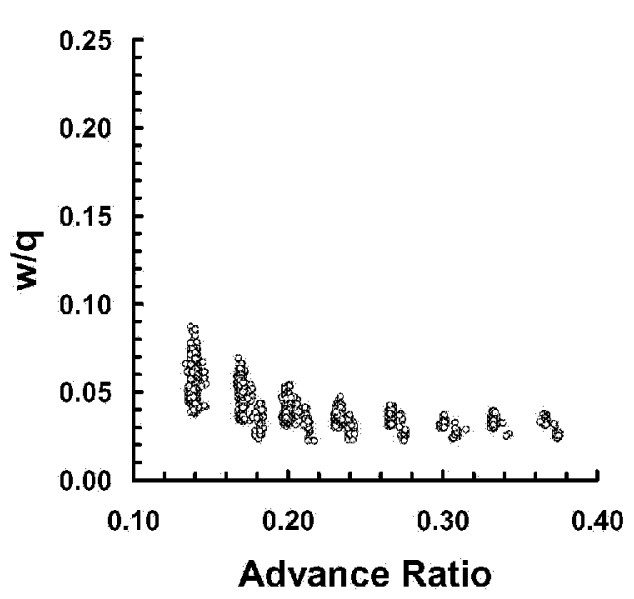

(a) Scatter data for microphone 1.

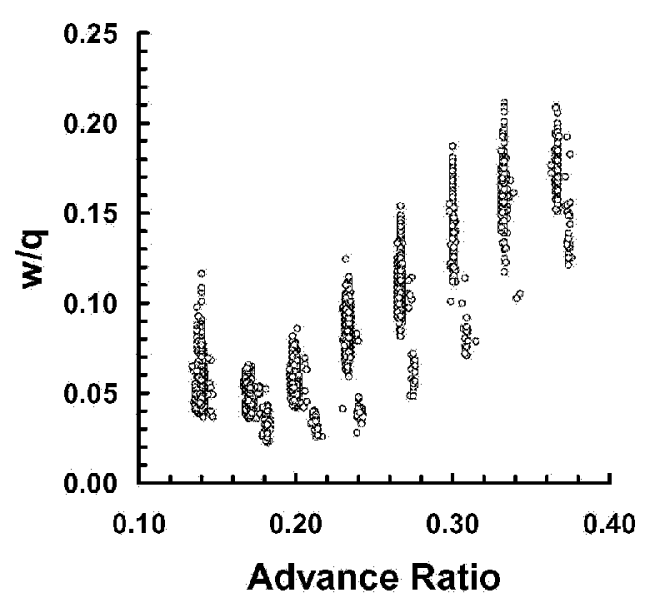

(b) Scatter data for microphone 4.

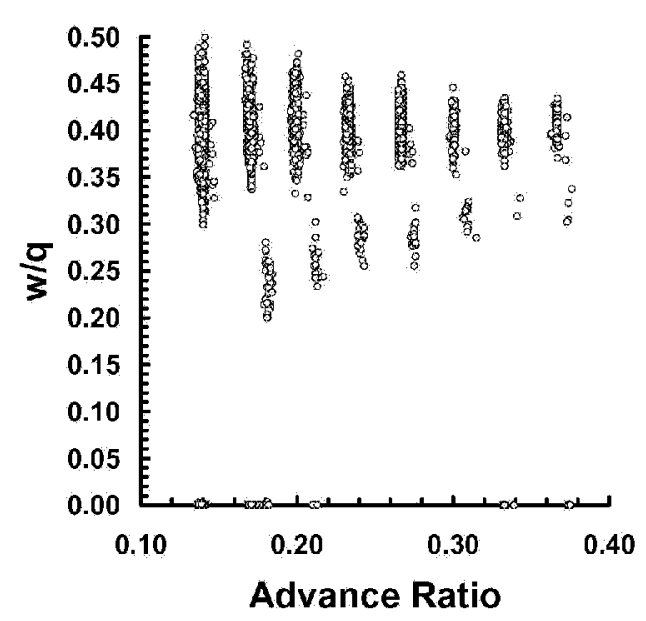

(c) Scatter data for microphone 6 .

Figure 6 - Microphone scatter data as a function of advance ratio for upstream microphones 1,4 , and 6 . 
data is most likely good. The trend is for $w / q$ to decrease with increasing tunnel speed at least between $\mu$ $=0.14$ and $\mu=0.17$. Then $w / q$ increases with tunnel speed, although the value is less than approximately 0.15 for all conditions up to and including $\mu=0.27$. However, the value of $\mathrm{w} / \mathrm{q}$ increases further for $\mu=0.30$ and above. Examination of spectral data for microphone 4 indicated that data up to and including $\mu$ $=0.27$ was acceptable, but data for $\mu=0.30$ and above was not acceptable. In figure 7 , spectra from microphones 1, 4, and 6 are compared at $\mu=0.27$. While the spectra from microphones 1 and 4 are similar, the spectra from microphone 6 displays a smoother spectra at a higher amplitude because the level of the non-harmonic noise is greater than the harmonic noise produced by the rotor. The upper bound for advance ratio for acceptable data appears to be $\mu=0.27$ for data measured with microphone 4.

Scatter data for microphone 6 are shown to be of a much higher level, even for low tunnel speeds and the trends remain approximately constant with increasing tunnel velocity, as shown in figure 6(c). This appears to indicate that data measured with microphone 6 will be unacceptable for all test conditions. The spectra shown from microphone 6 in figure 7 confirms this indication. Microphone 5 scatter data was similar to that for microphone 6. Perhaps in future tests, use of taller microphone stands at these locations will solve this problem, but the measurements obtained by microphones 5 and 6 are unusable for the current data set.

So, based on these results, the portion of the data set used for this study is limited to $\mu=0.27$ and below and uses microphone 4 as the estimate for the acoustic flux over the downstream control surface. Since BVI noise

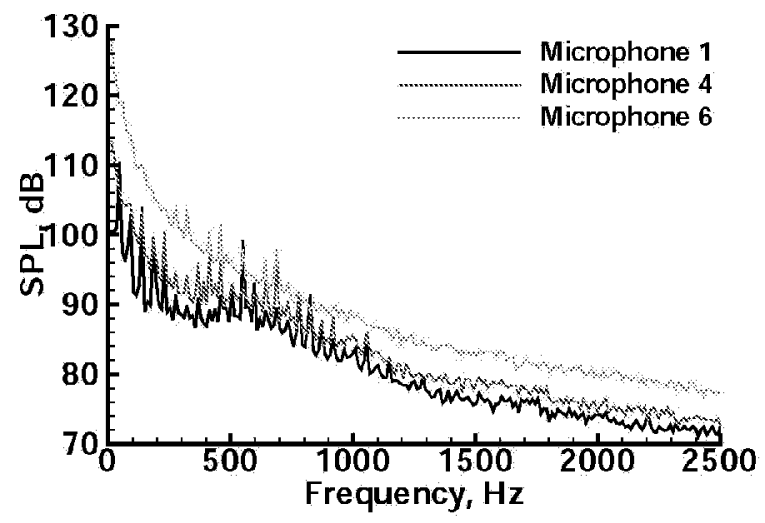

Figure 7. Spectra from microphones 1,4 , and 6 for $\mu=0.27$ and $\alpha=4 \mathrm{deg}$. is more prevalent in this low speed operating region, there should be no major impact from the advance ratio limitation. Resolution of the data quality issue allowed assessment of the noise characteristics of the ATR system to proceed.

Data Processing. In the previous study, the microphone spectra were all averaged to produce the sound pressure spectra that was then converted to sound power units by application of a transfer function. The transfer function was applied to all spectra and bandpass acoustic metrics were calculated. The difference in the level of the metrics were taken as the noise reduction numbers. A similar process was applied to the current data, except no effort was placed into determining a transfer function to put the sound pressure spectra into sound power units. The noise reduction results from this study should be directly comparable to the results from the previous study.

For the current study, the spectra were averaged on a power basis for each frequency bin for all 60 revolutions of data. This produced an averaged spectra for each microphone at each data point. The averaged spectra were then used to estimate the relative sound power levels for each rotor condition.

Since only one of the microphones downstream of the model produced a usable signal, the averaging process for the current study was weighted to give equal weight to the microphones upstream and the microphone down stream. The averaged spectra was then used to evaluate noise changes in the rotor model.

Acoustic metrics were computed for each operating condition using the acoustic energy in frequency bands representing low frequency thickness and loading noise and blade-vortex interaction noise. The frequency band chosen for the LFSPL, or low frequency sound pressure level, are from $0.5 \mathrm{f}_{\mathrm{bp}}$ to $6.5 \mathrm{f}_{\mathrm{bp}}$. The frequency band chosen for BVISPL, or blade-vortex interaction sound pressure level, was from $6.5 \mathrm{f}_{\mathrm{bp}}$ to $40.5 \mathrm{f}_{\mathrm{bp}}$. BVISPL and LFSPL levels were then used, respectively, to determine reduction in the BVI noise levels and the penalty incurred by any increases in the low frequency noise, reducing all of the acoustic data to just two numbers for each operating condition. The levels of these metrics can be compared between active-twist operating and not operating (baseline) conditions to examine acoustic trends due to active-twist control.

Obviously, the use of a single microphone measurement to estimate the downstream acoustic flux is not desirable and can be a source of error in the acoustic trends. This effect was quantified by recalculating the acoustic metric deltas, which are used to assess acoustic trends, 
using only a single upstream microphone. This calculation was performed for each of the three upstream microphones for the entire data set, and the resulting data were statistically analyzed to calculate the error. The difference in calculated delta metric levels using all three upstream microphones and only one upstream microphone was found to be approximately $0.5 \mathrm{~dB}$ for BVISPL and $0.7 \mathrm{~dB}$ for LFSPL. So the error band applicable to the noise level trends presented due to use of the single microphone downstream for BVISPL is $+/-0.5 \mathrm{~dB}$ and for LFSPL is $+/-0.7 \mathrm{~dB}$.

Vibration level is represented by the amplitude of the $4 \mathrm{P}$ component of the oscillatory fixed-system vertical shear force (4P NF). Although any number of metrics can be used to characterize vibration levels, it was felt that this parameter was probably the most relevant.

\section{Results}

A subset of operating conditions that allows evaluation of active-twist control effectiveness as a comprehensive noise and vibration reduction method can be obtained by considering only two conditions from each activetwist phase sweep. In order to determine the magnitude of BVI noise reduction, and assess any acoustic or vibration penalties, the active-twist control phase conditions for which BVISPL was minimized were chosen. Similarly, in order to assess any acoustic penalties incurred by maximum vibration reduction, the active-twist control phase conditions for which $4 \mathrm{P} \mathrm{NF}$ was minimized were chosen.

Accordingly, the results section is divided into two parts, first an examination the BVI noise reduction potential of the ATR system, and second, an examination acoustic consequences of best vibration reduction using active-twist. The results are plotted with the values for BVISPL, LFSPL, and 4P NF plotted as a function of $\alpha$ for each $\mu$. In all cases, the baseline case, which is the rotor operated without active-twist control, is plotted with both symbols and connecting line for clarity, while the values achieved by $3 \mathrm{P}, 4 \mathrm{P}$, and $5 \mathrm{P}$ active-twist control inputs at $1000 \mathrm{v}$ actuation voltage amplitude are plotted as symbols only.

\section{BVI noise reduction}

In figure 8 , BVISPL level as a function of $\alpha$ is examined for the advance ratio matrix. Two points are immediately apparent: in general, 5P active-twist control achieved lower BVISPL levels than 3P and 4P, and although most test conditions show some decrease in BVISPL, the magnitude of that reduction is rather small, usually on the order of $2 \mathrm{~dB}$ or less. At $\mu=0.14$, the difference between the effectiveness of the various control frequencies is most apparent, and even then the $4 \mathrm{P}$ control shows up to be nearly as effective as the $5 \mathrm{P}$ control, except where the BVISPL reduction is greatest at $\alpha=6$ and 7 degrees, where $4 \mathrm{P}$ data were not obtained. A maximum BVISPL reduction at $\alpha=6$ degrees was found to equal $2.8 \mathrm{~dB}$, followed by a 2.7 $\mathrm{dB}$ reduction at $\alpha=7$ degrees. At those conditions, 3P active-twist control is not as effective as $5 \mathrm{P}$ control in reduction of BVISPL. There are no corresponding $4 \mathrm{P}$ data for $\alpha=6$ and 7 degrees, conclusive statements about the effectiveness of $5 \mathrm{P}$ vs. $4 \mathrm{P}$ cannot be made with the current data set.

The magnitude of BVI noise reduction achieved by active-twist control is a bit disappointing, but is not as insignificant as it may appear at first. In the previous study $^{1}$, the maximum noise reduction achieved was 5.6 $\mathrm{dB}$ and that was achieved at $\mu=0.11$. The current study was limited to $\mu=0.14$ and above, due to tunnel speed limits that currently exist. The maximum control amplitude for the current and previous studies are both 1.5 degrees. If the results from the previous study from $\mu=0.14$ and above are considered, then the maximum noise reduction is between 3 and $4 \mathrm{~dB}$, which is only a $\mathrm{dB}$ or so greater than the reduction shown by the current data set. So active-twist control is nearly, but not quite, as effective as $\mathrm{HHC}$ in reduction of BVISPL. This statement is further supported by examination of the averaged spectra for the baseline condition and $5 \mathrm{P}$ active-twist control $1000 \mathrm{v}$ amplitude for $\mu=0.14, \alpha=$ $6 \mathrm{deg}$ as shown in figure 9. The reduction in the frequency range from $300 \mathrm{~Hz}$ to $900 \mathrm{~Hz}$ accounts for most of the BVISPL noise reduction shown in figure 8 (a). The trend shown in figure 9 is the same shown in figures 6 and $8(\mathrm{~b})$ of the previous study ${ }^{1}$. So the activetwist control shows similar, if smaller, magnitude reduction of BVISPL and a similar character in the spectral data comparison.

In the HHC studies ${ }^{1-3}$, it was found that increasing the amplitude of the control deflection increased the noise reduction. In figure 10 , the BVISPL increase relative to the baseline case is plotted as a function of control input phase for $5 \mathrm{P}$ active-twist at two actuation voltage amplitudes. One thing to notice is that for this case, active-twist control is equally adept at increasing and decreasing BVISPL level. Also, the change in BVISPL for the $1000 \mathrm{v}$ case at 320 degrees corresponds to the point plotted in figure $8(\mathrm{~b})$. It is interesting that the trends in BVISPL change are similar between the two actuation voltage cases, and it is apparent that the higher actuation amplitude provides the greater level of BVISPL reduction. Larger amplitudes of active-twist control blade tip deflection would probably provide more BVI noise reduction. 


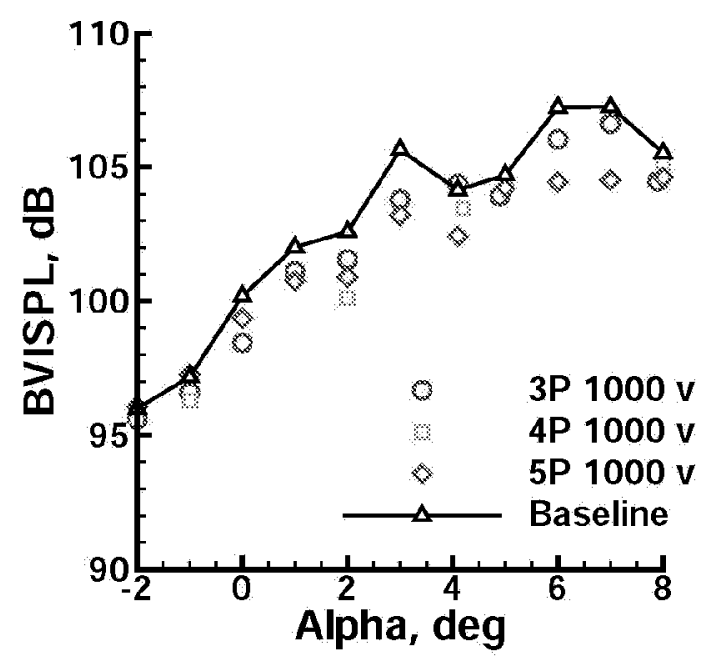

(a) $\mu=0.14$

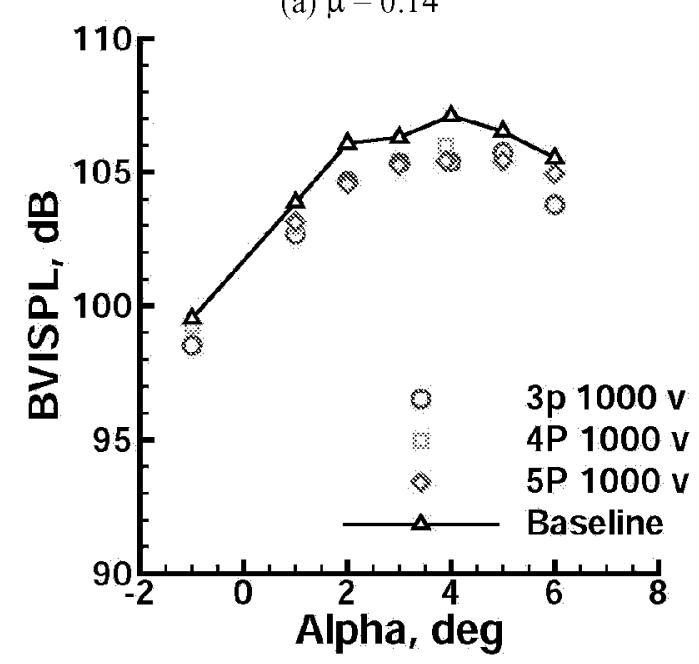

(b) $\mu=0.17$

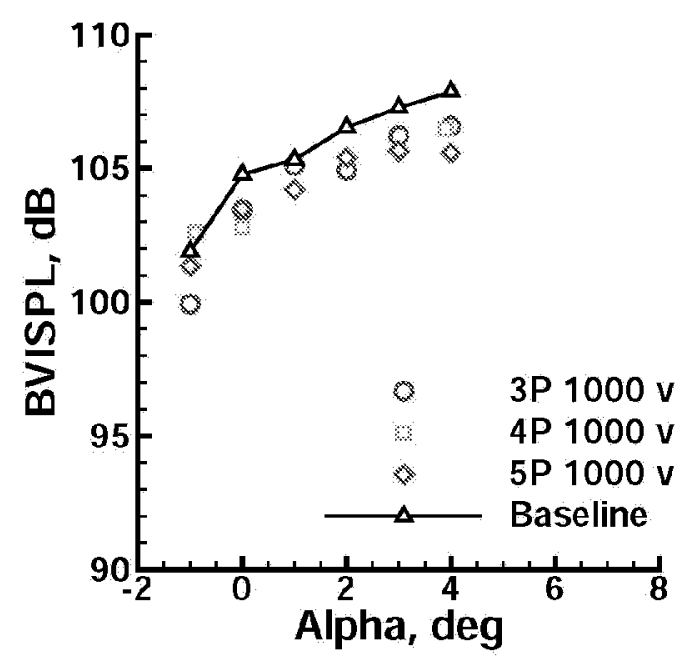

(c) $\mu=0.20$

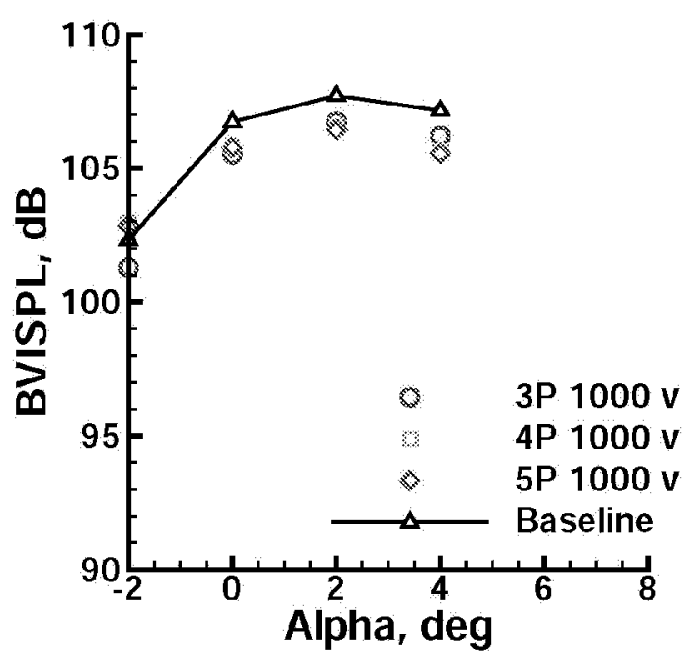

(d) $\mu=0.23$

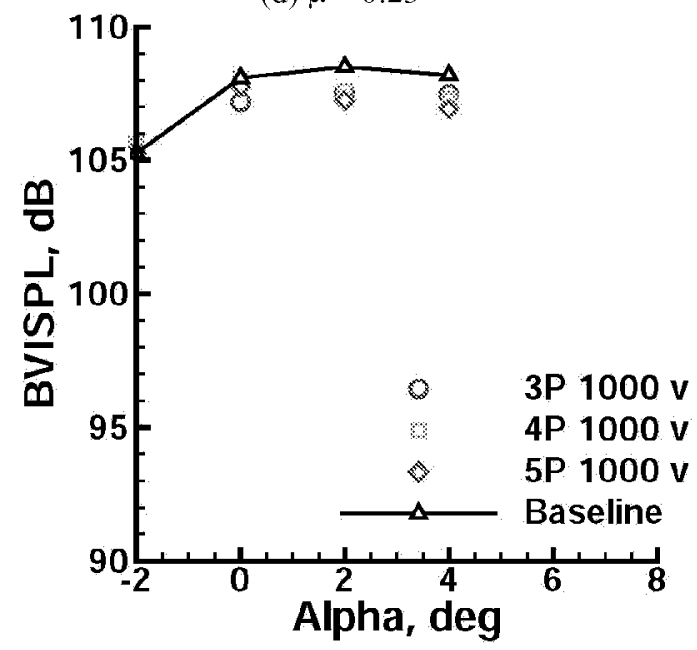

(e) $\mu=0.27$

Figure 8. BVISPL as a function of $\alpha$ for each advance ratio for minimum BVISPL conditions.

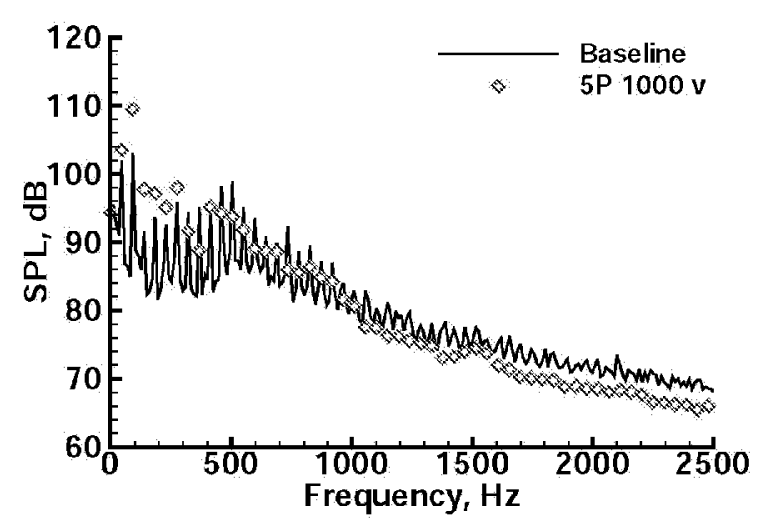

Figure 9. Spectral comparison between baseline and maximum BVI noise reduction case for $\mu=0.14$ and $\alpha=6$ deg. 


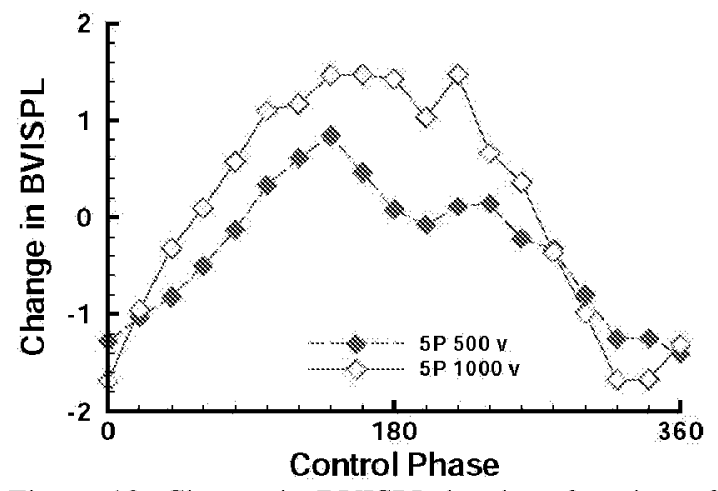

Figure 10. Change in BVISPL level as function of control phase for two amplitudes of 5P active-twist control for $\mu=0.17$ and $\alpha=4 \mathrm{deg}$.

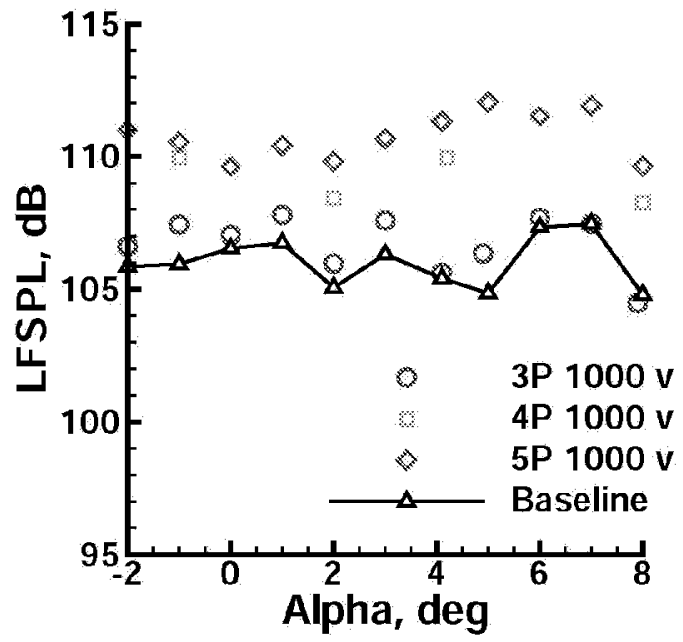

(a) $\mu=0.14$

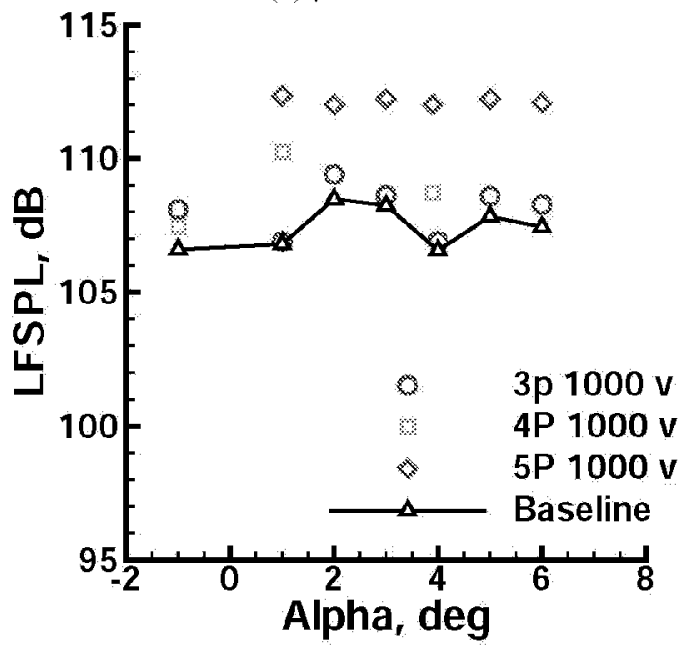

(b) $\mu=0.17$

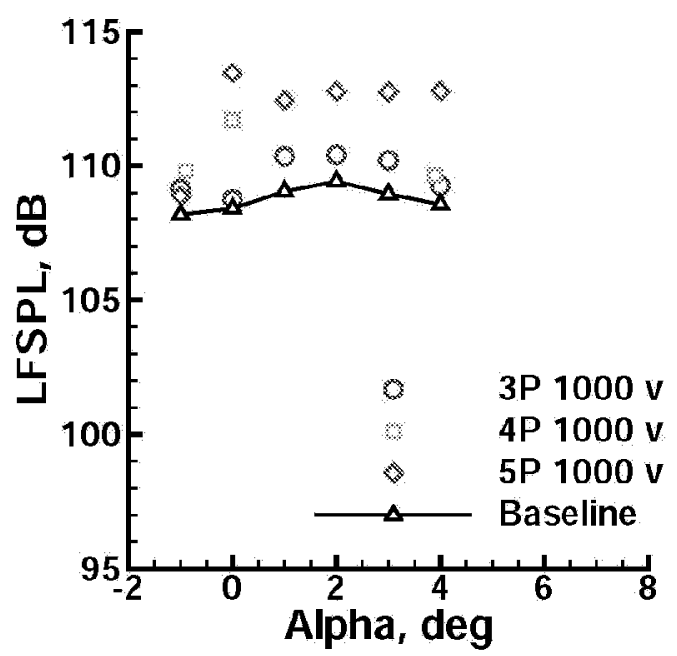

(c) $\mu=0.20$

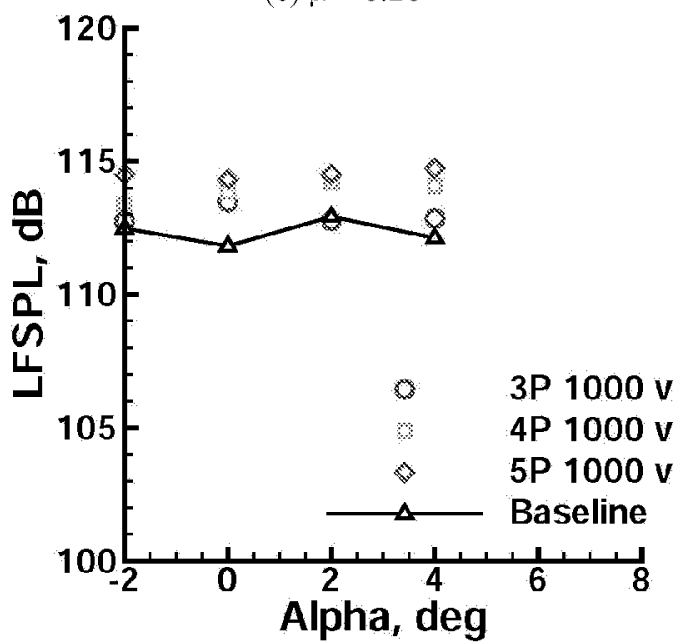

(d) $\mu=0.23$

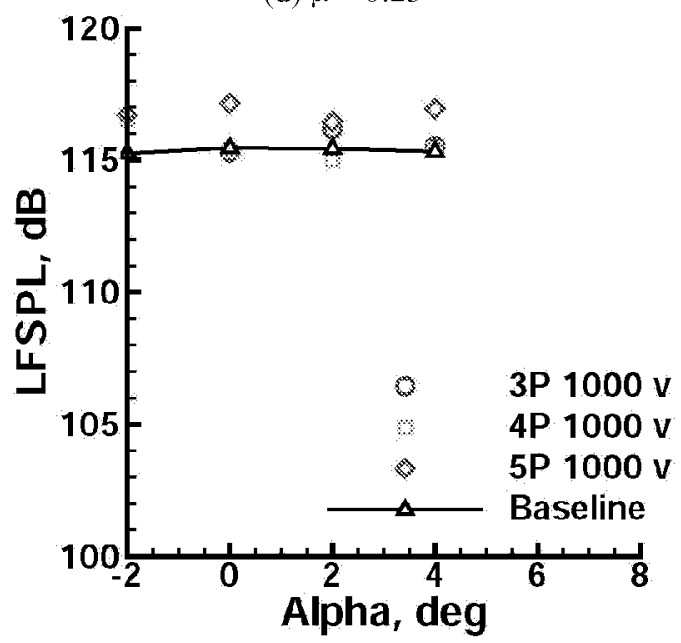

(e) $\mu=0.27$

Figure 11. LFSPL as a function of $\alpha$ for the minimum BVISPL case. 

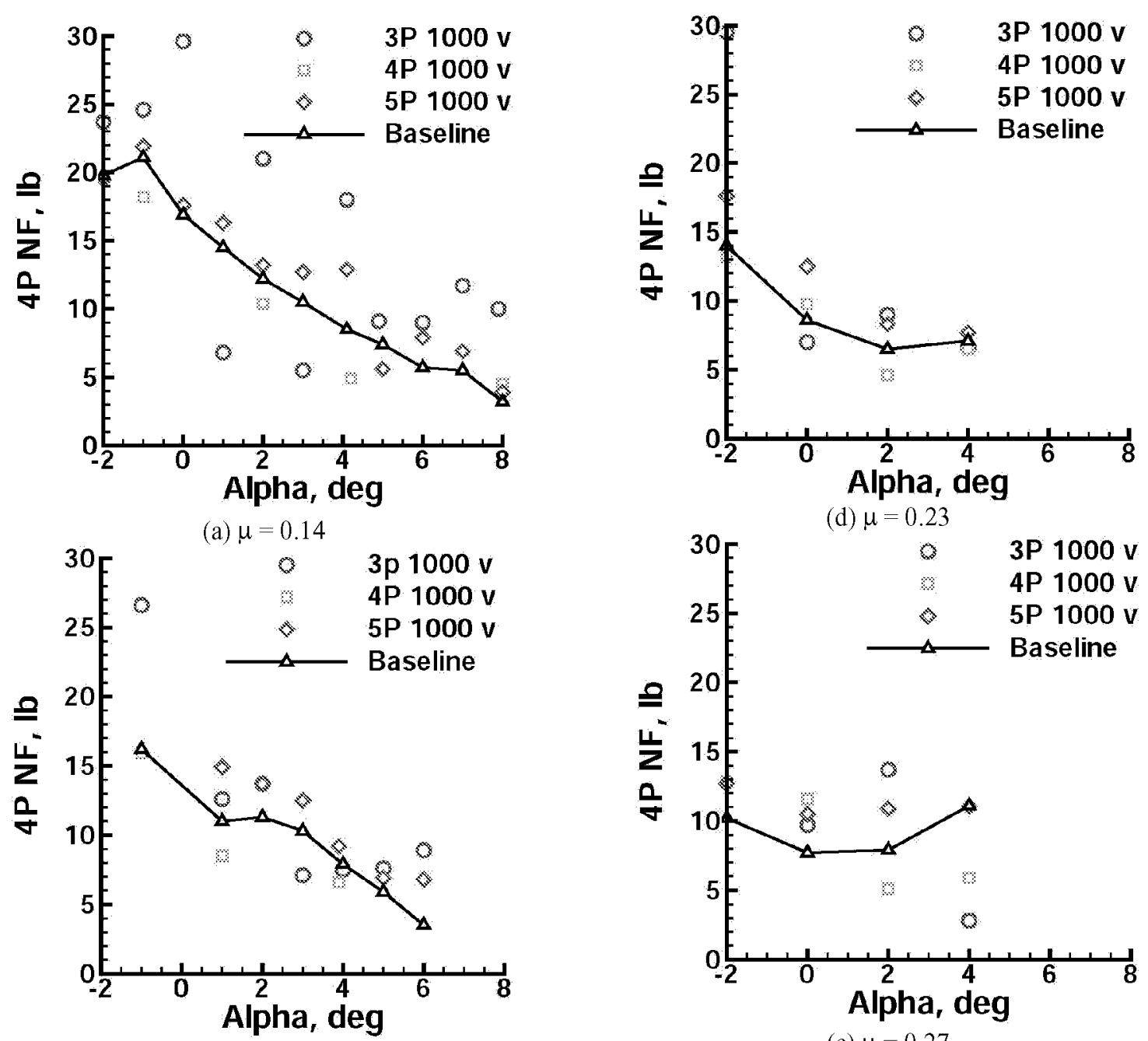

(b) $\mu=0.17$

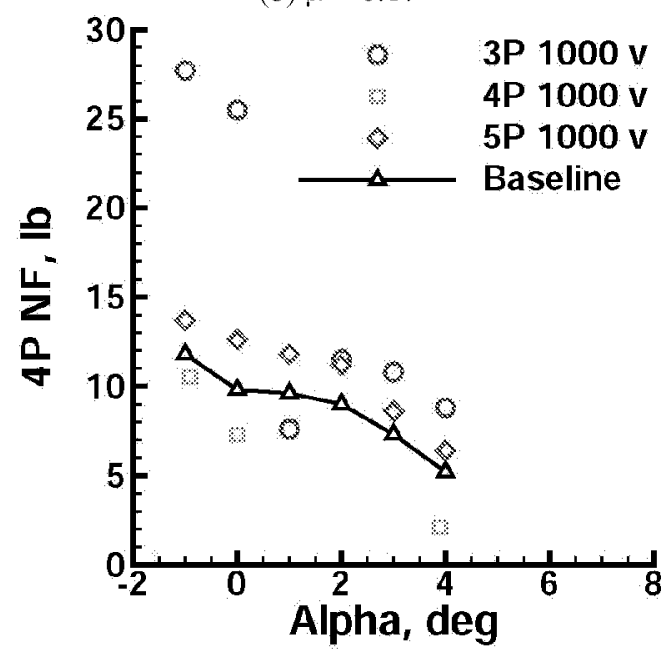

(e) $\mu=0.27$

Figure 12. 4P NF amplitude as a function of $\alpha$ for the minimum BVISPL case.

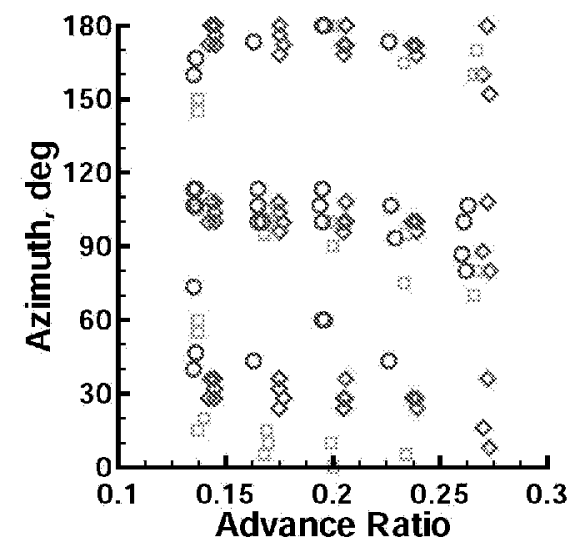

Figure 13. - Azimuth angle corresponding to maximum negative blade twist for maximum BVISPL reduction. Legend same as for figure 12 . 
Low Frequency Noise Level Increase due to BVI Noise Reduction. In figure 11, LFSPL corresponding to minimum BVISPL cases is plotted as a function of $\alpha$ for the advance ratio range. LFSPL is seen to increase for most of the test conditions, and that the increase was most apparent for 5P active-twist control. Correspondingly, the change in LFSPL for $3 \mathrm{P}$ activetwist control is fairly minimal, as is the reduction in BVISPL offered by $3 \mathrm{P}$ control settings. $5 \mathrm{P}$ control settings increased LFSPL most and this increase is greatest for $\mu=0.14$, where the largest BVISPL reductions were measured. However, the greatest LFSPL increase of $7 \mathrm{~dB}$ occurred at $\alpha=5$ degrees, while the maximum BVISPL reductions occurred at $\alpha=$ 6 and 7 degrees. Smaller LFSPL increases of $5 \mathrm{~dB}$ are also shown for $\mu=0.17$ and 0.20 in figures 11 (b) and 11(c), respectively. The LFSPL increase for this activetwist control system appears similar on trends to that shown by HHC in the earlier study ${ }^{1}$.

Vibration Level Effects of BVI Noise Reduction. In figure 12, the amplitude of the $4 \mathrm{P} \mathrm{NF}$ amplitude is plotted as a function of $\alpha$ for the advance ratio envelope. It is seen that $4 \mathrm{P} \mathrm{NF}$ amplitude is very sensitive to $3 \mathrm{P}$ active-twist control input. Large increases of 4P NF amplitude in response to 3P activetwist control input at $\mu=0.14$ in figure 12(a) correspond to minimal BVISPL decreases shown in figure $8(\mathrm{a})$. Conversely, while 5P active-twist control input was shown in figure $8(\mathrm{a})$ to produce the largest BVISPL decreases, the corresponding $4 \mathrm{P} \mathrm{NF}$ amplitude increases are shown to be relatively small increases. Although the $5 \mathrm{P}$ control input results in about as large an increase in 4P NF amplitude as 3P control over the rest of the advance ratio range, the $3 \mathrm{P}$ BVISPL decreases were less than those resulting from $5 \mathrm{P}$ control inputs. Hence the vibratory load penalty incurred by BVISPL reduction for $3 \mathrm{P}$ control input is higher than the penalty for $5 \mathrm{P}$ control input. It is also important to remember that the baseline amplitudes upon which this penalty is assessed are relatively low for this portion of the operating envelope.

Phase Angle for Minimum BVI noise. If the BVI noise reduction mechanism is the same for all frequencies of active-twist control tested, then there should be some relationship between the control phases that produce the noise reduction. One of the BVI noise reduction mechanisms proposed for $\mathrm{HHC}$ is to reduce the strength of the vortex as it is released from the blade. With active-twist control, this would probably closely correspond with the maximum negative (nose down) twist. Using the control phase for all the minimum BVI cases, and calculating the azimuthal locations of the maximum negative twist for 3,4 , and
5P yields a set of azimuth locations for each advance ratio. If the same physical event causes all the frequencies to reduce noise, then the azimuth points from the different frequencies of control actuation should tend to overlay. In figure 13, azimuth points corresponding to the maximum negative twist for active-twist control phase angles that produced BVI noise reduction are presented as a function of advance ratio. It is seen that the points for all three frequencies overlay along a line extending from an azimuth angle of 110 degrees at $\mu=0.14$ to an azimuth angle of 90 degrees at $\mu=0.27$. This is the portion of the azimuth where a blade vortex important for BVI noise might be released. This result may prove useful in formulation of a control law for BVI noise reduction using active-twist control.

A corresponding correlation was not observed on the retreating side of the rotor disk as might be expected to occur for retreating side BVI events. One possible explanation is that the advancing side BVI noise events may be sufficiently louder to dominate the overall acoustic signal in this reverberant environment. Further measurements in an anechoic environment may well reveal a similar set of phase angles for reduction of retreating side BVI noise.

\section{Vibration reduction}

While BVISPL noise reduction was shown to incur both an increase in LFSPL and 4P NF amplitude, conversely, the operation of active-twist control to obtain minimum 4P NF amplitude can have adverse consequences for BVISPL and LFSPL. Accordingly, the data base was queried for the control phase angle resulting in minimum 4P NF amplitude. Resulting data are presented in figures 14-16.

In figure 14, BVISPL is shown to be affected, but not always adversely by the control settings that showed the lowest vibration levels. In fact, the data points showing the largest BVISPL reduction in figure 8(a) are also shown here in figure 14(a). This is an indication that conditions where BVISPL and 4P NF amplitude are simultaneously reduced are possible. In general, BVISPL is not largely affected by operation for minimum 4P NF amplitude, so active-twist control for minimum vibration will not incur a large BVISPL penalty.

In figure 15, LFSPL is shown to be increased for activetwist control conditions yielding minimum $4 \mathrm{P} \mathrm{NF}$ amplitude as much as LFSPL was increased for minimum BVISPL operation. The maximum LFSPL penalty shown is $7 \mathrm{~dB}$, just as in figure 11 . 


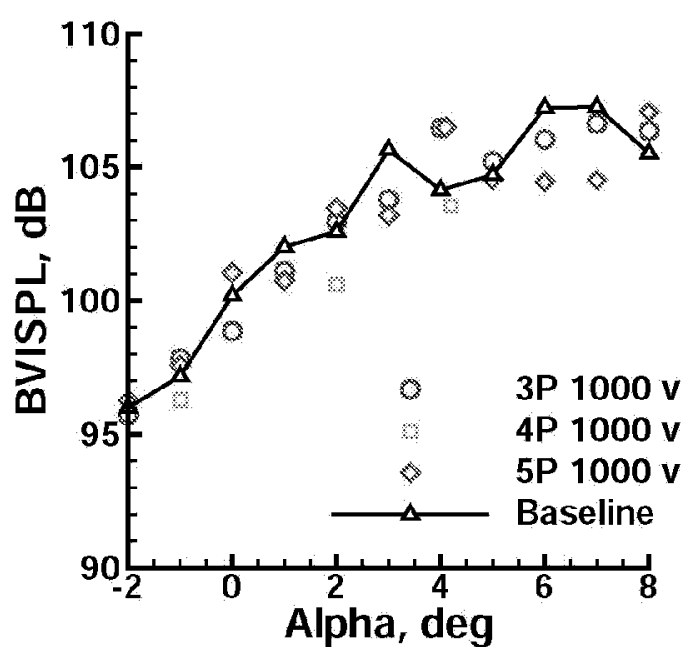

(a) $\mu=0.14$

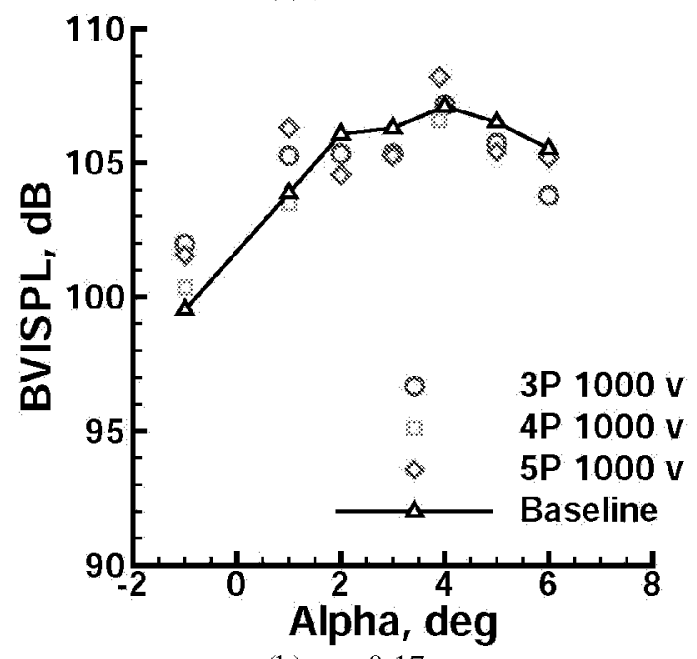

(b) $\mu=0.17$

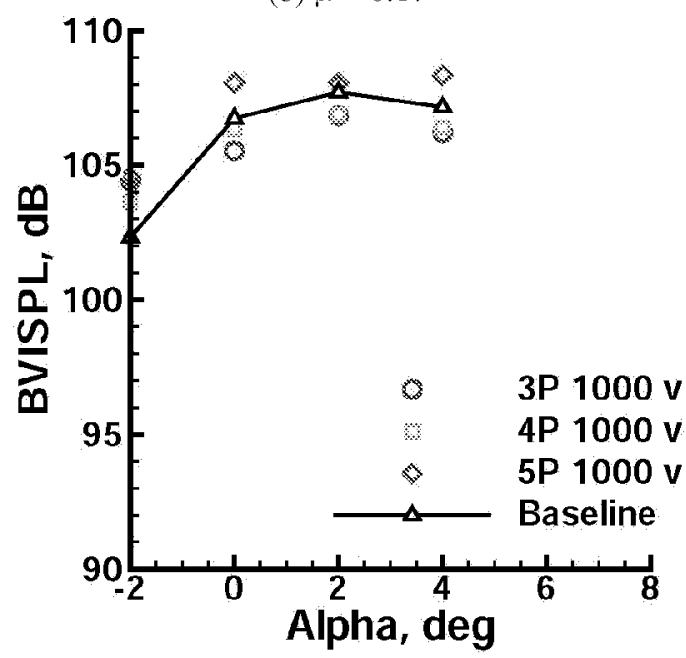

(c) $\mu=0.20$

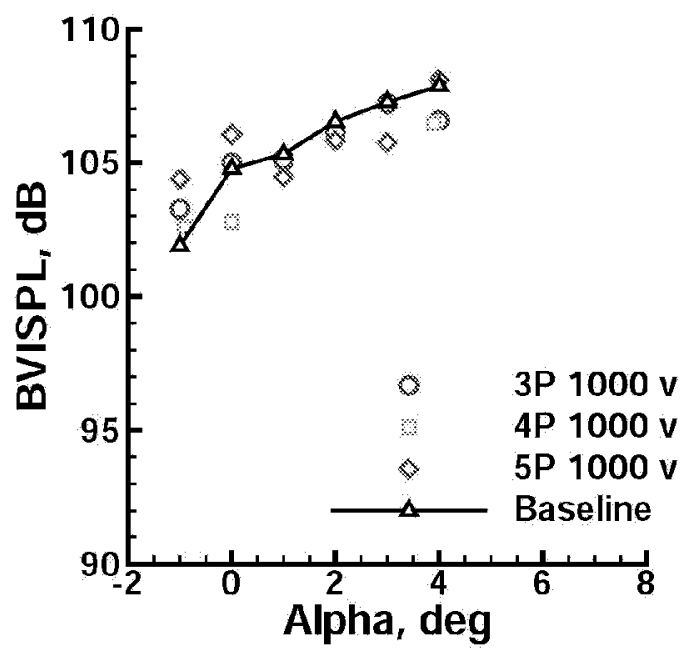

(d) $\mu=0.23$

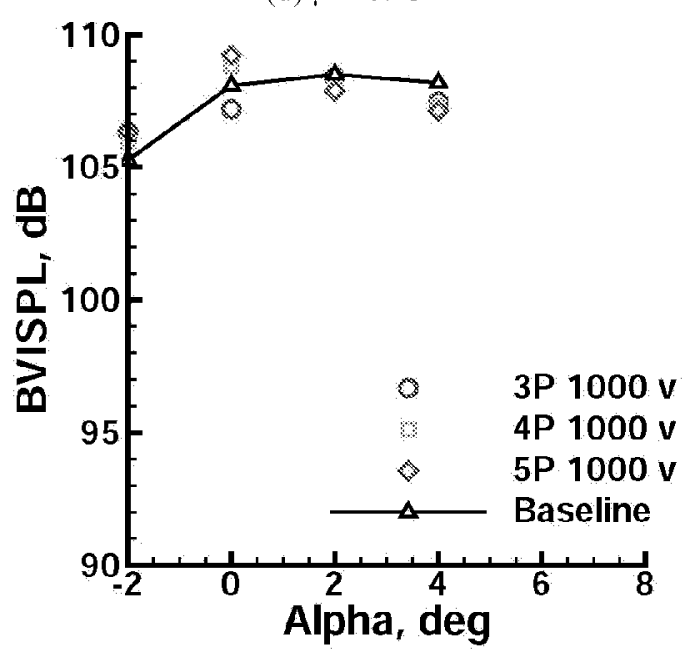

(e) $\mu=0.27$

Figure 14. BVISPL as a function of $\alpha$ for the minimum 4P NF amplitude case. 


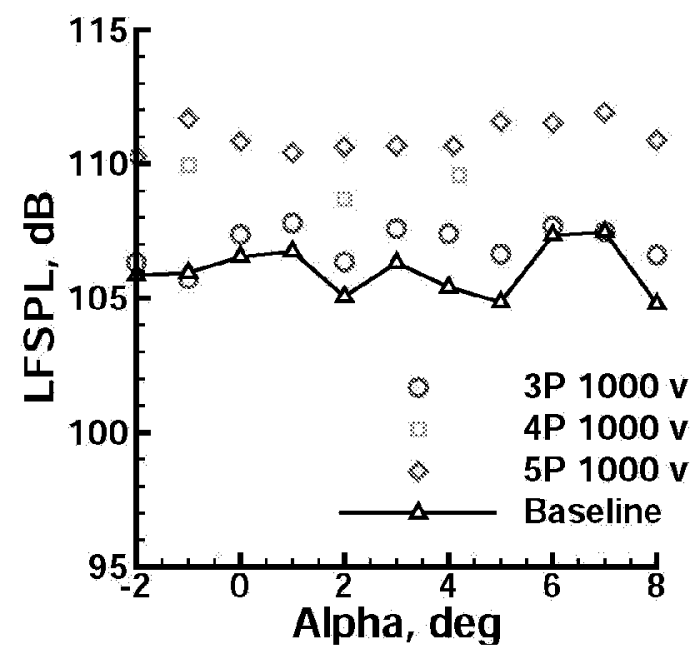

(a) $\mu=0.14$

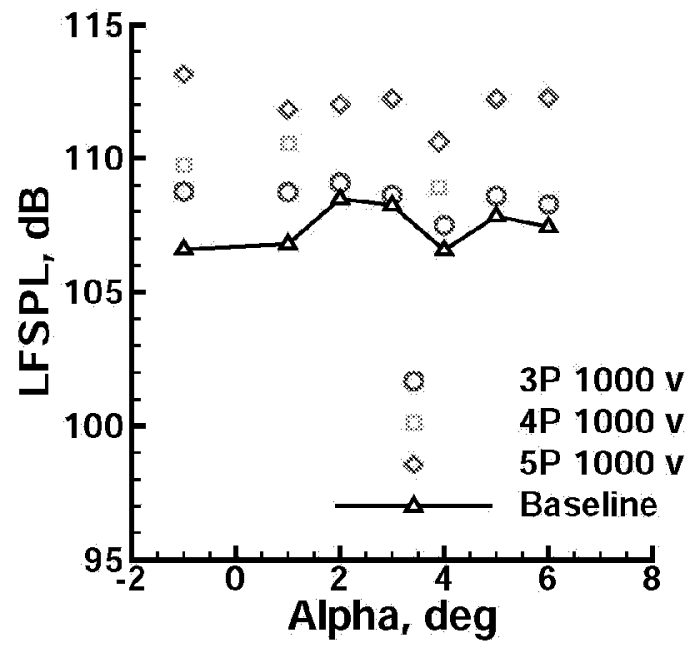

(b) $\mu=0.17$

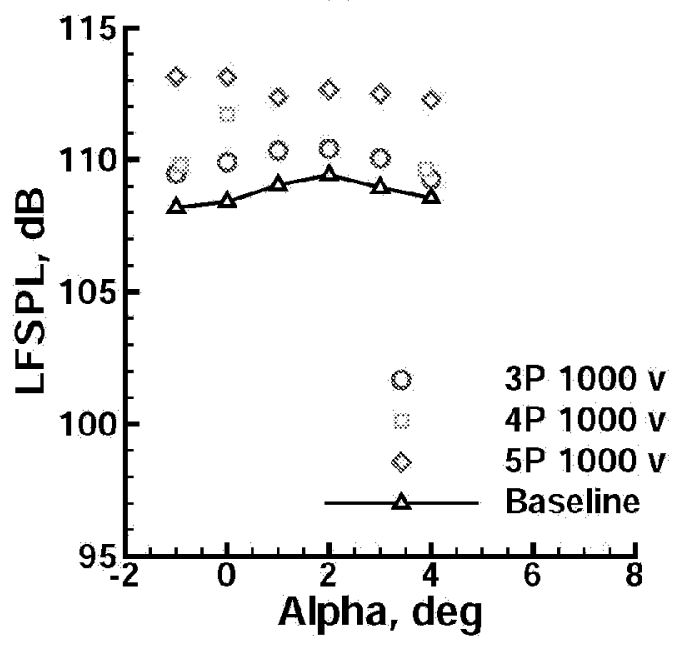

(c) $\mu=0.20$

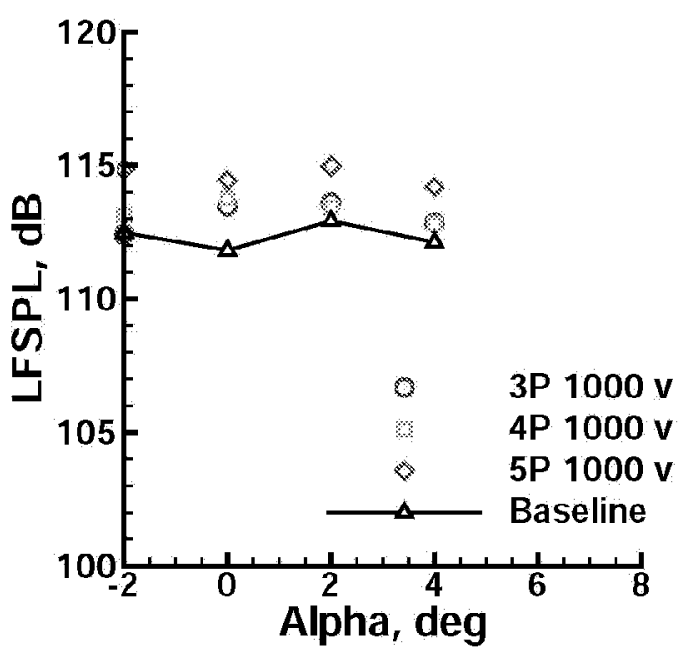

(d) $\mu=0.23$

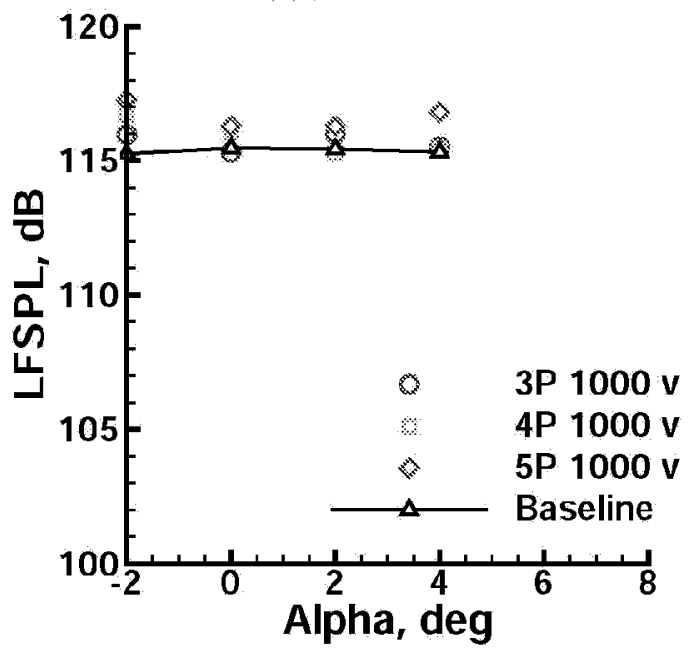

(e) $\mu=0.27$

Figure 15. LFSPL as a function of $\alpha$ for the minimum 4P NF amplitude case. 


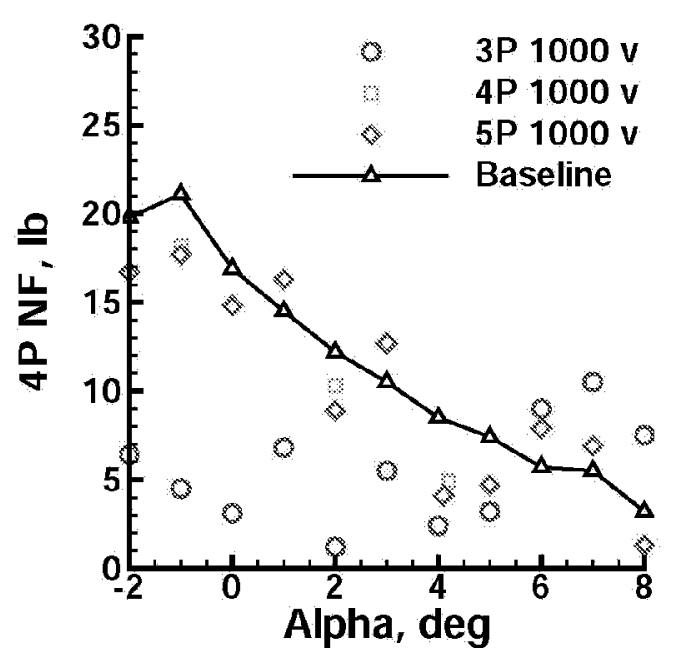

(a) $\mu=0.14$

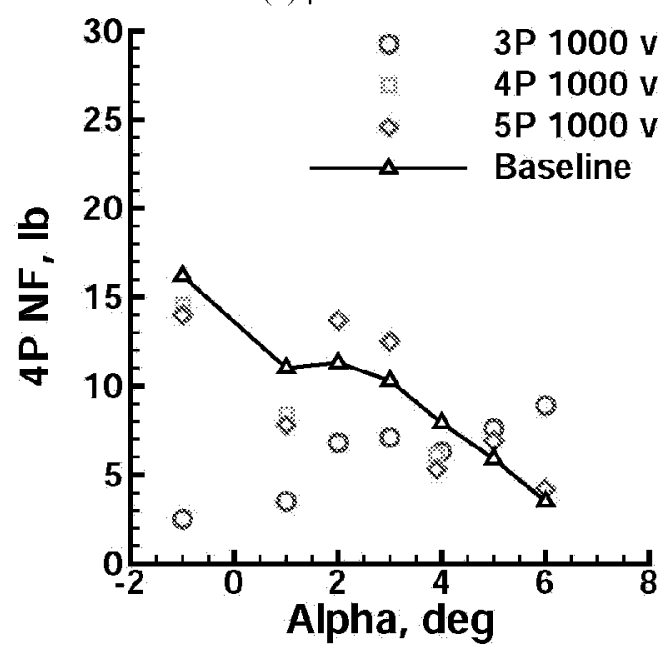

(d) $\mu=0.17$

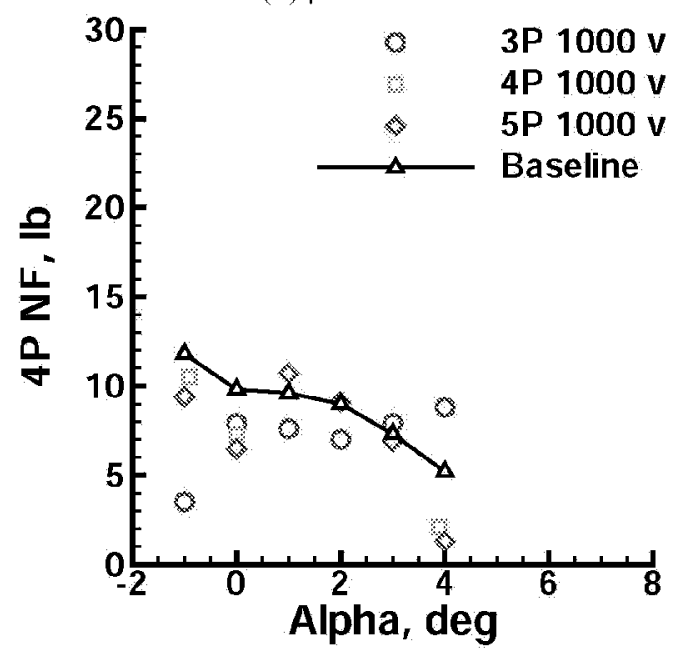

(c) $\mu=0.20$

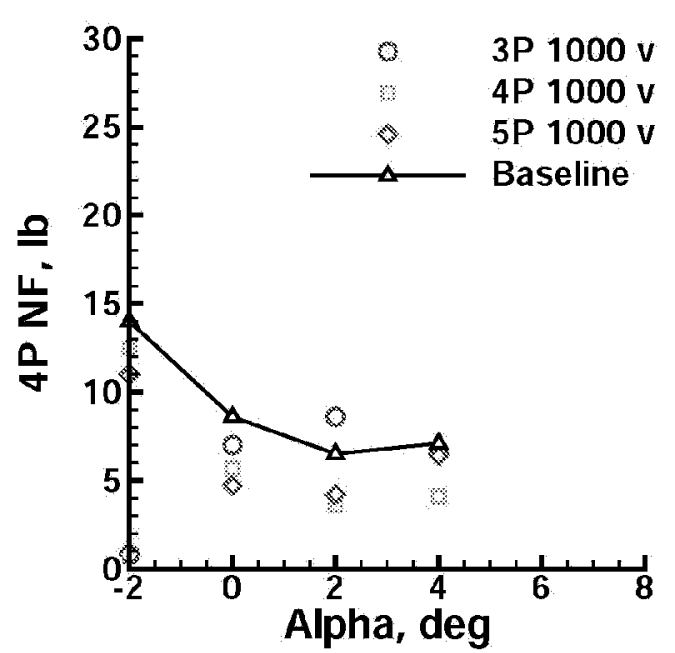

(d) $\mu=0.23$

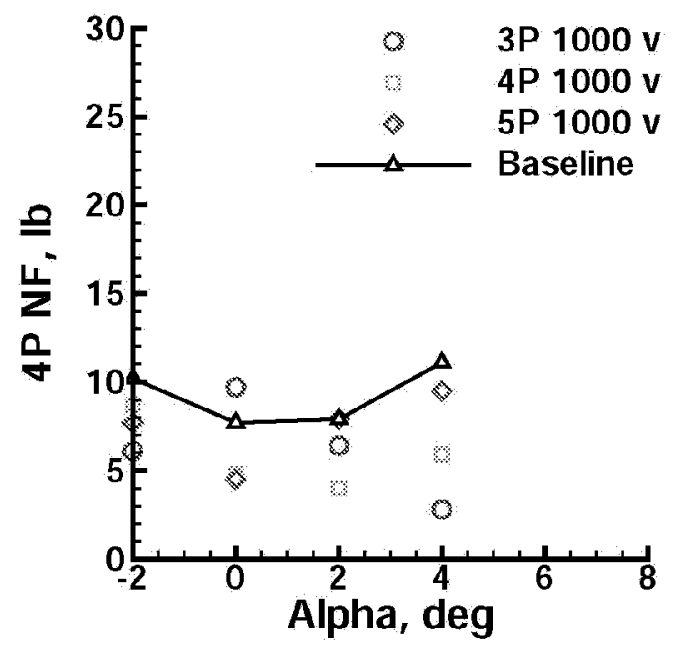

(e) $\mu=0.27$

Figure 16. 4P NF amplitude as a function of $\alpha$ for the minimum 4P NF amplitude case. 
In figure 16 , it is seen that, in general, lower $4 \mathrm{P} \mathrm{NF}$ amplitudes are achievable using the $3 \mathrm{P}$ control input as opposed to the $4 \mathrm{P}$ and $5 \mathrm{P}$ control inputs. This result is confirmed in references 6 and 8 .

\section{Conclusions}

An initial test of active-twist control in open-loop operation in which fixed system oscillatory loads were measured simultaneously with acoustic data has been conducted in the Transonic Dynamics Tunnel.

The largest BVISPL noise reduction found was $2.8 \mathrm{~dB}$. BVISPL was found to be most sensitive to $5 \mathrm{P}$ operation. The amplitude of the BVISPL reduction was consistent with, if a bit smaller than, an earlier study with $\mathrm{HHC}$ on the same rotor test stand in the same facility.

LFSPL penalties for the BVISPL reduction were found to be up to $7 \mathrm{~dB}$ at some conditions. $4 \mathrm{P}$ NF amplitude was affected by the conditions that decreased BVISPL, although both increases and decreases were found.

Conditions selected to minimize 4P NF amplitude showed that BVISPL was affected, but both increases and decreases were found. LFSPL penalties for the minimum vibration case were generally as severe as for the minimum BVI case. The $3 \mathrm{P}$ control input was generally most effective at achieving lower $4 \mathrm{P} \mathrm{NF}$ amplitude.

Active-twist control is probably not quite as effective in reducing BVISPL as $\mathrm{HHC}$, but it probably provides better vibration reduction. Active-twist control is an excellent vibration reduction technology and it may be possible to add some BVI noise reduction capability to this system by using multiple frequency control input, perhaps employing $5 \mathrm{P}$ control for noise reduction while using $3 \mathrm{P}$ control for vibration reduction. Increased blade deflection control authority will probably benefit BVI noise reduction capabilities of active-twist control.

\section{References}

1. Brooks, T. F.; Booth, E. R., Jr.; "The Effects of Higher Harmonic Control on Blade-Vortex Interaction Noise and Vibration", Journal of the American Helicopter Society, Vol. 38 (3), July 1993.

2. Splettstoesser, W. R., Schultz, K. J., Kube, R., Brooks, T. F., Booth, E. R., Nies1, G., Streby, O., "A Higher Harmonic Control Test in the DNW to Reduce Impulsive BVI Noise," Journal of the American Helicopter Society, Vol. 39, (4), October 1994.
3. Kube, R., Splettstoesser, W. R., Wagner, W., Seelhorst, U., Yu, Y. H., Tung, C., Beaumier, P., Prieur, J., Rahier, G., Spiegel, P., Boutier, A., Brooks, T. F., Burley, C. L., Boyd, D. D., Mercker, E., Pengel, K., HHC Aeroacoustic Rotor Tests in the German Dutch Wind Tunnel: Improving Physical Understanding and Prediction Codes. American Helicopter Society $52^{\text {nd }}$ Annual Forum, Washington, D. C., June 1996.

4. Swanson, S. M., Jacklin, S. A., Blaas, A., Kube, R., Niesl, G., "Individual Blade Control Effects on Blade-Vortex Interaction," American Helicopter Society $50^{\text {th }}$ Annual Forum, Washington, D.C., May 1994.

5. Marcolini, M. A., Booth, E. R., Tadghighi, H., Hassan, A. A., Smith, C. D., Becker, L. E., "Control of BVI Noise using an Active Trailing Edge Flap," American Helicopter Society Vertical Lift Aircraft Design Conference, San Francisco, CA, January 1995.

6. Wilbur, M. L., Mirick, P.H., Yeager, W. T., Jr., Langston, C. W., Cesnik, C. E. S., Shin, S, "Vibratory Loads Reduction Testing of the NASA/Army/MIT Active Twist Rotor," American Helicopter Society $57^{\text {th }}$ Annual Forum, Washington D.C., May 2001.

7. Fleming, G. A., Soto, H. L., South, B. W., "Projection Moiré Interferometry for Rotorcraft Applications: Deformation Measurements of Active Twist Rotor Blades," American Helicopter Society International $58^{\text {th }}$ Forum, Montréal, Canada, June 2002.

8. Wilbur, Matthew L., Yeager, William T., Jr, and Skula, Martin K., "Further Examination of the Vibratory Loads Reduction Results from the NASA/Army/MIT Active Twist Rotor Test," American Helicopter Society $58^{\text {th }}$ Annual Forum Proceedings, Montréal, Canada, June 2002. 